\title{
Zeolite Thin Film-Coated Fiber Sensors Based on Fabry-Perot Interferometer for Detection of Chemical Vapors
}

\author{
Xiangping NING, Chunliu ZHAO*, Feifei SHI, and Juan KANG \\ Institute of Optoelectronic Technology, China Jiliang University, Hangzhou, 310018, China \\ *Corresponding author: Chunliu ZHAO_E-mail: zhchunliu@homail.com
}

\begin{abstract}
A novel zeolite-coated fiber sensors for detection of volatile organic compounds (VOCs) based on the Fabry-Perot interferometer was proposed and demonstrated. The sensor comprised a polycrystalline silicalite thin film grown up on the cleaved end face of a standard single-mode fiber. The inline Fabry-Perot cavity was composed by the end face of the single-mode fiber and the thin film. The sensor device operated by measuring the interference signal, which was a function of the amount of chemical vapor adsorption in its crystalline micro porous structure. Experimental results showed that the proposed VOC sensor worked well and the sensitivities were $2.78 \times 10^{-3} \mathrm{~dB} / \mathrm{ppm}$ when the concentration ranged from $350 \mathrm{ppm}$ to $2100 \mathrm{ppm}$ and $1.23 \times 10^{-3} \mathrm{~dB} / \mathrm{ppm}$ when the concentration ranged from $2100 \mathrm{ppm}$ to $5250 \mathrm{ppm}$.
\end{abstract}

Keywords: Zeolite film, volatile organic compound (VOC) sensor, Fabry-Perot interferometer

Citation: Xiangping NING, Chunliu ZHAO, Feifei SHI, and Juan KANG, "Zeolite Thin Film-Coated Fiber Sensors Based on Fabry-Perot Interferometer for Detection of Chemical Vapors,” Photonic Sensors, 2015, 5(2): 137-141.

\section{Introduction}

The detection of volatile organic compounds (VOCs) has been found useful in many areas including environmental pollutant monitoring, food quality assurance, and emission control [1]. In recent years, the sensors based on optical fibers have been attracting increasing interest due to their advantages such as the small size, remote measurement, and immunity to electromagnetic interference.

Various sensors have been demonstrated by optical fiber Fabry-Perot (F-P) interferometer sensors. The F-P cavity can be constituted by a section of the fiber between two dielectric mirrors [2] or a gap between two dielectric end surfaces [3]. An interferometer signal which is formed by two reflections at two end surfaces is a function of the length and refractive index of the cavity. As a result, a fiber F-P sensor is capable of measuring various parameters which are resulted from environmental changes. Because of the high sensitivity and quick response time, the fiber F-P sensors are particularly attractive for applications involving pressure and temperature measurement [4].

Zeolites are microporous aluminosilicate crystals framed by uniform subnanometer-scale or nanometer-scale pore systems. The nonporous zeolites can selectively adsorb molecules due to the size exclusion or shape selectivity which depends primarily on the crystal structure, framework $\mathrm{Si} / \mathrm{Al}$ ratio, and type of extra framework cations. Zeolites are thermally and chemically more stable than the polymer cladding of an optical fiber. In recent decades, zeolite membranes and thin films have

Received: 30 November 2014/ Revised version: 23 January 2015

(C) The Author(s) 2015. This article is published with open access at Springerlink.com

DOI: $10.1007 / \mathrm{s} 13320-015-0233-4$

Article type: Regular 
been demonstrated for applications in chemical vapors detection [5], chemical sensors for the detection of dissolved organics in water [6] and measurement of trace chemicals [7]. However, keeping the high sensitivity with a wide measurement range is still a problem.

In this paper, a novel zeolite-coated fiber sensor for the detection of the VOCs based on the Fabry-Perot interferometer is proposed and demonstrated. Isopropanol was chosen as a model chemical because of its nontoxic nature. The interference intensity of the Fabry-Perot interferometer would change with its surrounding isopropanol concentration and sense the absorption for organic molecules of the silicalite. Experimental results showed that the proposed sensor for chemical vapor measurement worked well and the sensitivities of the isopropanol concentration were $2.78 \times 10^{-3} \mathrm{~dB} / \mathrm{ppm}$ when the concentration ranged from $350 \mathrm{ppm}$ to $2100 \mathrm{ppm}$ and $1.23 \times 10^{-3} \mathrm{~dB} / \mathrm{ppm}$ when the concentration ranged from $2100 \mathrm{ppm}$ to $5250 \mathrm{ppm}$.

\section{Experimental setup}

The sensor was made by growing the thin silicalite film on the cleaved end face of a standard single-mode fiber by in situ crystallization from an aluminum-free precursor solution using tetrapropylammonium ion (TPA + ) as the structure directing agent (SDA). We prepared the synthesis solution by mixing $11.3 \mathrm{ml}$ of TPAOH (tetrapropylammonium hydroxide), $20.4 \mathrm{ml}$ of TEOS (tetraethyl orthosilicate), and $60 \mathrm{ml}$ of $\mathrm{H}_{2} \mathrm{O}$ [1]. Due to its low alkalinity and high organic concentration, this particular precursor was chemically friendly to the fiber surface. The mixture was vigorously stirred at $50^{\circ} \mathrm{C}$ for $4 \mathrm{~h}$. The hydrothermal synthesis was conducted at $180^{\circ} \mathrm{C}$ under the autogenous pressure for $4 \mathrm{~h}$. After the hydrothermal synthesis, the zeolite-coated fiber was washed with deionized water and dried in air at $500{ }^{\circ} \mathrm{C}$ for $2 \mathrm{~h}$ to remove the TPA SDA. Figure 1 shows the optical microscopic image of a silicalite-film-coated fiber. The thickness of the zeolite film was about $20 \mu \mathrm{m}$, and the thickness would increase with the addition of hydrothermal synthesis time. The zeolite was constituted by zeolite crystals in cuboid particles which had straight channels with a diameter of about $6 \AA[6]$. So the end face of the zeolite film was uneven.
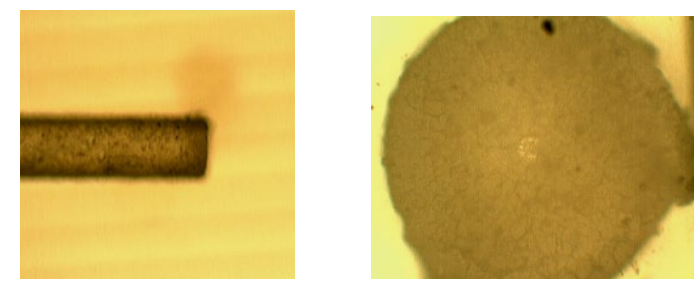
fiber.

Fig. 1 Optical microscopic image of a silicalite-film-coated

Figure 2 shows the experimental setup of the proposed F-P interferometer-based optical fiber sensors for chemical vapor measurement. The chemical vapor detection is based on the measurement of the adsorption-induced effective optical length of the F-P cavity. The wavelength of interference peak will change with the optical length of the F-P cavity, and the thickness of the F-P cavity is the same as the thickness of the film which is about $20 \mu \mathrm{m}$. The light from a broadband light source (BBS) is launched into a single-mode optical fiber and split into two paths through a $3-\mathrm{dB}$ fiber coupler. One of the paths is angle cleaved to eliminate back reflection. The other path is spliced to the sensor probe, as illustrated in the enlarged view. An optical spectrum analyzer (OSA) is used to monitor the reflection from the F-P cavity.

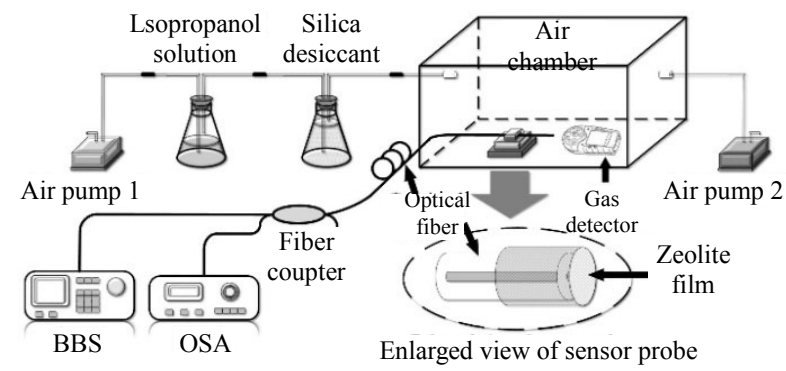

Fig. 2 Experimental setup of the proposed chemical vapor sensor. 
The fiber sensor probe was placed in a vapor chamber made of plexiglass. The length, width, and height of the chamber was $70 \mathrm{~cm}, 40 \mathrm{~cm}$, and $30 \mathrm{~cm}$, respectively. The concentration of the isopropanol in the chamber was varied by adjusting the air flow rate which was controlled by the air pump 1 and air pump 2. The isopropanol concentration of the gas flowing through the isopropanol solution accommodated Erlenmeyer flask would increase with the accelerated rate of gas flow in the air pump 1 and decrease with the accelerated rate of gas flow in the air pump 2. The silica desiccant would absorb water vapor mixed in the gas flowing into the air chamber. The isopropanol concentration in the air chamber was detected and recorded by the gas detector. All of the reported experiments were conducted at the room temperament $\left(21^{\circ} \mathrm{C}\right)$ under the atmospheric pressure.

The nonporous zeolites can selectively adsorb the molecules in the straight channels among zeolite crystals. With the adsorption of chemical vapor molecules, the refractive index will change. Therefore, the optical path is varied, and the wavelength of interferometry peaks shifts. Furthermore, an increase in the reflectivity at the interface of the zeolite film is owing to an increase in the refractive index, which enhances the reflected intensity of the F-P cavity. The detection of the concentration can be achieved by the measurement of the wavelength shift and reflectedintensity.

\section{Results and discussions}

Figure 3 shows the interference spectra in response to the isopropanol concentration. The interferometer intensity of the spectra from $1500 \mathrm{~nm}$ to $1590 \mathrm{~nm}$ changed about $10 \mathrm{~dB}$, and the interference peaks shifted $10 \mathrm{~nm}$ towards the longer wavelength with the concentration of isopropanol increasing from $0 \mathrm{ppm}$ to $5250 \mathrm{ppm}$. Such changes in intensityis resulted from an increase in the zeolite film reflectivity included the increasing sorption of organic molecules in the zeolite cavity. At the same time, the refractive index of the thin film increased with the addition of the gas concentration, which increased the optical path difference. So the wavelength of interferometry peaks shifted with the isopropanol concentration. Furthermore, some tiny interference deeps accompanied with the interference spectra. These interference deeps were caused by the unevenness of the end face of the thin film and the air pockets in the zeolite film.

As shown in Fig. 3, half of the free spectrum range (FSR) was from $1535 \mathrm{~nm}$ to $1550 \mathrm{~nm}$, and $\Delta \lambda_{\mathrm{FSR}}=30 \mathrm{~nm}$. The refractive index of the zeolites film was around 1.33 [6]. So the length of the F-P cavity $L$ was $29 \mu \mathrm{m}$, which conformed to the measured value of $20 \mu \mathrm{m}$.

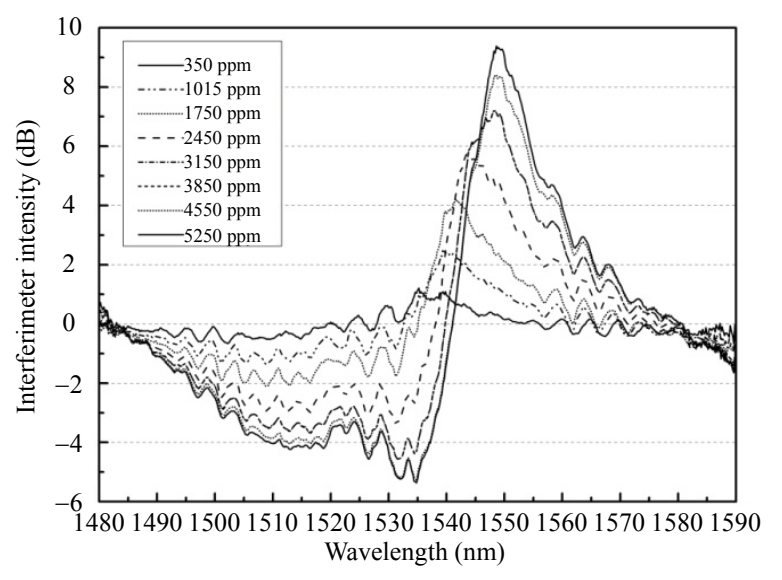

Fig. 3 Spectra of the interference intensity when the concentration is in the range of $350 \mathrm{ppm}$ to $5250 \mathrm{ppm}$.

Figure 4 shows the relationship between the wavelength shift of the interference peaks and concentration. The interference peaks shifted from $1539 \mathrm{~nm}$ to $1548 \mathrm{~nm}$ when the concentration of isopropanol increased from $350 \mathrm{ppm}$ to $2450 \mathrm{ppm}$. The fitting function is $y=4.28 \times 10^{-3} x+1537.58$, and the fitting degree was 0.9981 . The concentration sensitivities for the demodulation of the wavelength was $4.28 \times 10^{-3} \mathrm{~nm} / \mathrm{ppm}$. The OSA with a $20-\mathrm{pm}$ measurement precision was used in our experiments, so the concentration precision could reach about $4.7 \mathrm{ppm}$. The wavelength of the interference peaks was saturated when the concentration was more than $2450 \mathrm{ppm}$. 


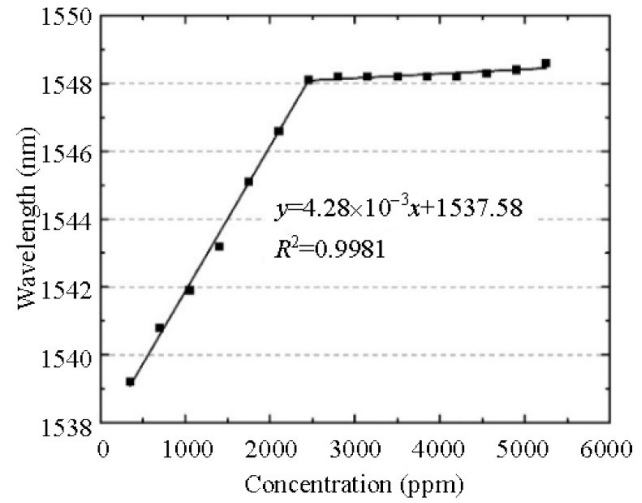

Fig. 4 Relationship between the wavelength shift of the interference peaks and concentration.

Figure 5 shows the relationship between the interference intensity and concentration which increased from $0 \mathrm{ppm}$ to $5250 \mathrm{ppm}$ with a step of 350 $\mathrm{ppm}$. The curve at the top portion of the figure is the intensity of the interference peaks. The intensity was monotonically increased in the concentration range from $350 \mathrm{ppm}$ to $5250 \mathrm{ppm}$, and an inflection point existed at $1400 \mathrm{ppm}$. When the concentration was below $1400 \mathrm{ppm}$, the fitting function is $y=4.28 \times 10^{-3} x-0.469$. The sensitivities reduced but still kept a well linearity when the concentration was above $1400 \mathrm{ppm}$ and the fitting functions is $y=9.43 \times 10^{-4} x+4.157$. The experimental data fitted very well with the linear functions with the fitting degree of 0.9979 and 0.9947 , respectively. The sensitivities for the demodulation were $4.28 \times 10^{-3} \mathrm{~dB} / \mathrm{ppm}$ when the concentration ranged from $350 \mathrm{ppm}$ to $1400 \mathrm{ppm}$ and $9.43 \times 10^{-4} \mathrm{~dB} / \mathrm{ppm}$ when the concentration ranged from $1400 \mathrm{ppm}$ to $5250 \mathrm{ppm}$.

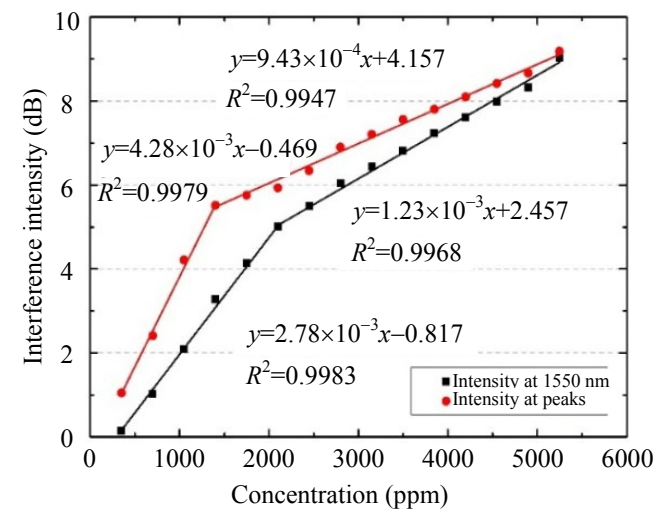

Fig. 5 Relationship between the interference intensity and concentration.
The intensities of the interference peaks were susceptible to the tiny interference deeps accompanied with the interference spectra. That easily caused the deviation of the intensity. So we considered the intensity at a specific wavelength.

Experimental data in the bottom of Fig. 5 showed that the intensity at $1550 \mathrm{~nm}$ had the different linear relationship when the concentration was below and above $2100 \mathrm{ppm}$. The experimental data fitted well with the linear functions with the fitting degree of 0.9983 and 0.9968 , respectively. The fitting functions are $y=2.78 \times 10^{-3} x-0.817$ and $y=1.23 \times 10^{-3} x+2.457$. The sensitivities for the demodulation of $1550 \mathrm{~nm}$ were $2.78 \times 10^{-3} \mathrm{~dB} / \mathrm{ppm}$ in the concentration range of $350 \mathrm{ppm}$ to $2100 \mathrm{ppm}$ and $1.23 \times 10^{-3} \mathrm{~dB} / \mathrm{ppm}$ in the concentration range of $2100 \mathrm{ppm}$ to $5250 \mathrm{ppm}$.

The sensitivity of the demodulation of $1550 \mathrm{~nm}$ was a little lower than those of the interference peaks when the concentration was below the inflection point. However, above $2100 \mathrm{ppm}$, the sensitivity was better than that of the demodulation of interference peaks. Furthermore, the fitting degree was higher than that of the demodulation of interference peaks. The OSA with a $0.01-\mathrm{dBm}$ measurement precision was used in our experiments, so the precision could reach about $3.60 \mathrm{ppm}$ in the concentration range of $350 \mathrm{ppm}$ to $2100 \mathrm{ppm}$ and $8.13 \mathrm{ppm}$ in the concentration range of $2100 \mathrm{ppm}$ to $5250 \mathrm{ppm}$.

The sensor in our work could keep a high sensitivity in a wide concentration which ranged from $350 \mathrm{ppm}$ to $5250 \mathrm{ppm}$. Compared with [5], we achieved an improvement of 15 times in the sensitivity. Additionally, despite the higher sensitivities in [6,7], the measuring range could only keep in dozens ppm. Meanwhile, we could keep a relatively high sensitivity in the concentration range of $350 \mathrm{ppm}$ to $5250 \mathrm{ppm}$.

\section{Conclusions}

A novel zeolite-coated fiber sensors for the 
detection of volatile organic compounds based on the Fabry-Perot interferometer is proposed in this paper. The sensor operated by monitoring the change in the interference intensity of the F-P cavity. With its simple structure, the sensitivities were $2.78 \times 10^{-3} \mathrm{~dB} / \mathrm{ppm}$ when the concentration ranged from $350 \mathrm{ppm}$ to $2100 \mathrm{ppm}$ and $1.23 \times 10^{-3} \mathrm{~dB} / \mathrm{ppm}$ when the concentration ranged from $2100 \mathrm{ppm}$ to $5250 \mathrm{ppm}$.

\section{Acknowledgement}

This work was supported by the International Technological Cooperation Program of Zhejiang Province, China.

Open Access This article is distributed under the terms of the Creative Commons Attribution License which permits any use, distribution, and reproduction in any medium, provided the original author(s) and source are credited.

\section{References}

[1] H. Xiao, J. Zhang, J. Dong, M. Luo, R. Lee, and V.
Romero, "Synthesis of MFI zeolite films on optical fibers for detection of chemical vapors," Optics Letters, 2005, 30(11): 1270-1272.

[2] C. Wu, H. Y. Fu, K. K. Qureshi, B. Guan, and H. Y. Tam, "High-pressure and high-temperature characteristics of a Fabry-Perot interferometer based on photonic crystal fiber," Optics Letters, 2011, 36(3): 412-414.

[3] C. Liao, S. Liu, L. Xu, C. Wang, Z. Wang, Z. Li, et al., "Sub-micron silica diaphragm-based fiber-tip Fabry-Perot interferometer for pressure measurement," Optics Letters, 2014, 39(10): 2827-2830.

[4] T. Wei, Y. Han, H. Tsai, and H. Xiao, "Miniaturized fiber inline Fabry-Perot interferometer fabricated with a femtosecond laser," Optics Letters, 2008, 33(6): $536-538$.

[5] H. Xiao, J. Zhang, J. Dong, M. Luo, R. Lee, and V. Romero, "Synthesis of MFI zeolite films on optical fibers for detection of chemical vapors," Optics Letters, 2005, 30(11): 1270-1272.

[6] J. Zhang, X. Tang, J. Dong, T. Wei, and H. Xiao, "Zeolite thin film-coated long period fiber grating sensor for measuring trace chemical," Optics Express, 2008, 16(11): 8317-8323.

[7] J. Zhang, J. Dong, M. Luo, H. Xiao, S. Murad, and R. A. Normann, "Zeolite-fiber integrated optical chemical sensors for detection of dissolved organics in water," Langmuir, 2005, 21(19): 8609-8612. 